\title{
WAYS OF REFERENCE TO MEINONGIAN OBJECTS \\ Ontological Commitments of Meinongian Theories
}

\section{CONTENTS}

\author{
1. Introduction \\ 2. Contemporary Formal Accounts \\ 3. A New Idea Concerning the Syntax \\ 4. First System of Meinongian Objects \\ 5. Ontological Commitments \\ 6. Second and Third System of Meinongian Objects \\ 7. Ten Magic Rings
}




\section{Introduction*}

A. Meinong's views are usually associated with an highly inflated ontology including various kinds of nonexistent objects, incomplete and impossible ones among others. Around the turn of the century B. Russell strongly criticised this ontology accusing it of inconsistency. And perhaps because of this criticism Meinong's views have been forgotten for a long time. Only recently some philosophers have created theories of objects which are formalisations of Meinong's ontology or which are directly inspired by the ontology ${ }^{1}$. However there still remains a need to discuss the problem of ways of reference to Meinongian objects regarding their specific structure of predication, and the interrelated problem of ontological commitment of those theories.

Meinong's ontological theory seems to be not so bizzare if we interpret it as a theory of possible intentional objects, i.e. possible objects of consciousness. And this interpretation perfectly agrees with phenomenological tradition from Brentano to Husserl and with the contemporary philosophy of mind. Needless to say reference is a special form of intentional relation so semantical investigations into these objects are of great relevance for general theory of intentionality.

Phenomenological or analytical descriptions reveal a very intriguing ontological feature of intentional objects. They have a double structure of predication which manifests particularly clear in nonexistent objects. For every such an object we can distinguish two groups of its properties: internal and external ones. Internal properties or qualities are those through which an object appears to consciousness. External properties are those which the object exemplifies when taken in the status of intentional object qua intentional. It is hard to explain more precisely the sense of this distinction without carrying out a complicated phenomenological analyses. But we hope it will be enough here to give paradigmatic examples. Let us mention two famous nonexistent objects and list some of their internal and external properties.

object

the round square internal properties

being round, being a square, (?) being a geometrical figure external properties

being impossible, being incom-

plete, being nonexistent, being thought of by J. Paśniczek, etc.

\footnotetext{
* Section headings introduced by the editors.

1 Cf. [4], [11], [12], [13], [14], [6], [3]. See also [5].
} 


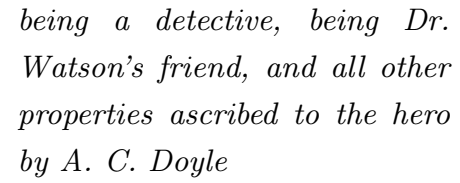

being a fictional character, being incomplete, being inconsistent, etc.

An intentional object may be incomplete or impossible only with respect to its internal properties whereas its external qualification is like any existent individual, i.e. externally the object is always possible and complete. There are two main conceptions of theoretical interpretation of internal-external distinction. According to the first, all properties are divided into nuclear and extranuclear ones: internal properties of an object are always nuclear, while external properties of an object are always extranuclear. According to the second conception, the conception of two modes of predication, an object possesses in different way its internal and external properties - we talk about the internal and the external predication respectively (the internal-external status of property may vary from object to object). Accordingly, we can distinguish in every intentional objects two subjects: the internal and the external one respectively (this may be considered as a third conception but I treat it as a supplementary to the second one). Perhaps, the conception of property distinction is closer to Meinong's original views and nowadays is followed by Parsons, Routley, and Jacquette. The conception of two modes of predication is rather credited to Meinong's student E. Mally and, independently, was put forward by Polish phenomenologist R. Ingarden. To-day the conception is advocated by E. Zalta and myself. We are not going to discuss the two conceptions in a more detailed way while comparing them with respect to their theoretical power or with respect to their adequacy as a theoretical explication of intentionality phenomena (some claim that these conceptions are formally equivalent ${ }^{2}$ ). Let us remark only that even if the first conception is adopted then one gets inevitably involved to some extent in the second conception as well. That is why, in what follows, we will consider the conception of two modes of predication.

\section{Contemporary Formal Accounts}

Among contemporary formal theories inspired by Meinong's views Parsons' and Zalta's are certainly best known and developed to the highest degree. They are strong second order intensional systems based on the classical logic and which, besides usual abstraction axioms for relations and properties,

\footnotetext{
${ }^{2}$ See [2].
} 
contain abstraction axioms for objects. Here we focus on Zalta's system as a system based on the conception of two modes of predication. The abstraction axiom takes the following form here (we omit some restrictions originally imposed on the axiom):

ABO $\exists x \forall P(x P \equiv \alpha) \quad(x$ does not occur free in $\alpha)$

The formula $x P$ expresses the internal predication and this the only syntactical novelty in the language of this system which distinguishes it from the classical language. In particular, the external predication is expressed by the classical formula $P x$. The $\mathbf{A B O}$ can be understood in this way that to every class of properties there correspond an object - we say that this class constitute the object. And two objects are identical iff they are constituted by the same properties. As an example let me define the famous nonexistent object the golden mountain:

$$
\exists x \forall P(x P \equiv P=\text { goldness } \vee P=\text { mountainhood })
$$

This objects is constituted by two properties: goldness, mountainhood. What is particularly important for us is that in Zalta's system we quantify objects both as subjects of internal and as subjects of external predication, i.e. both $\forall x(\ldots x P \ldots)$ and $\forall x(\ldots P x \ldots)$ are formulas of the system. So, taking seriously Quinean criterion according to which quantification is the strongest, if not the only, mean of reference, this system is ontologically committed to objects of Meinongian kind. It should emphasised that the system in question is also committed to properties and relations and not only because these are values of variables but because their existence is posited explicitly by the following definition axiom (we give here only a simplified version for properties):

ABP $\exists P \forall x(P x \equiv \alpha) \quad(P$ does not occur free in $\alpha)$

Unfortunately $\mathbf{A B O}$ and $\mathbf{A B P}$ together are inconsistent. To see this we can define the following antonymous object and property:

(I) $\quad a P \equiv P=Q, \quad$ where

(II) $Q x \equiv \forall P(x P \supset \neg P x)$

Notice that from (I) it easily follows:

$$
a Q
$$


Let us assume that:

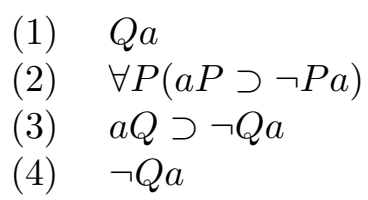

(from III and 3)

Now let us assume that:

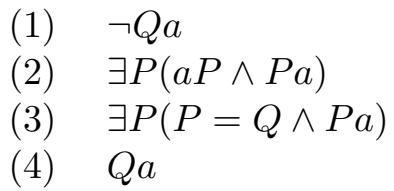

So we get $Q a \equiv \neg Q a .^{3}$ Does it mean that Russell was right after all? Zalta avoids this paradox and safes his theory at the cost of imposing some counterintuitive restrictions on $\mathbf{A B O}$ and $\mathbf{A B P}$. We can make some other ad hoc stipulations similar to that which block paradoxes in higher-order logics and set theories. However none of them seems to be acceptable from the phenomenological point of view. The only remedy which seems to be phenomenologically well grounded is the following: we cannot prevent an object to possess internally more properties than it is constituted of (actually if we think of object such that $P$ we ascribe to it many other properties different than $P$ ). Consequently we should assume such a definition axiom for objects which allow objects to possess all properties entailed, in a certain sense of the word, by its constituting properties. In other words, internal properties of objects must be closed under some appropriate entailment. As one can easily notice, this particular paradox will not go through when such an assumption is adopted. ${ }^{4}$ But, as we will see later on, this way avoiding paradoxes in Meinongian theories is not a universal one.

\footnotetext{
3 This paradox may be called the Clark paradox since Romane Clark was the first who presented a paradox arising in theories of objects of consciousness (originally his paradox pertains to the H. N. Castañeda's theory). Cf. [1].

4 There are good reasons to think that Parsons' and Zalta's abstraction axioms for objects do not properly explain how we 'create' or think objects in our consciousness (i.e. these axioms are unacceptable from the phenomenological point of view). Let us turn back to the definition of the golden mountain. What is defined by this definition, we claim, is the $\mathrm{M}_{1}$-object constituted by the property of being gold, being mountain and being nothing else. By and such, every object of consciousness is constituted by a finite number of properties (it is according to phenomenological analyses of consciousness) but this does not preclude that an object may possess infinitary many properties (some objects may possess infinitary many properties entailed by the finitary many properties constituting the objects).
} 
Now the problem is whether it is really necessary to be involved in such a strong existential object commitment (not to mention the commitment to properties and relations) and, even worse, to be endangered seriously by inconsistency. We are asking this question not because of reluctance to Meinongian objects as existentially very dubious kind of entities (if they are entities at all!). We are just wondering whether there can be still another, in a sense weaker, way of reference to Meinongian objects and, consequently, weaker ontological commitments. Since there are two modes of predication associated with Meinongian objects we can quantify these objects as subjects of only one mode or both modes of predication. This gives rise to several possible ways of reference. Obviously, every such a way of reference must be built in a formal language and a formal theory of Meinongian objects i.e. a theory which captures if not all than at least the most important principles of Meinongian object ontology.

Before we proceed further, let us point out to some essential distinction which is frequently obscured when the Quine's criterion of ontological commitment is taken into account. There are many versions of this criterion so different from each other that we should rather talk about different criterions. Generally, two main Quine's criterions of ontological commitment can be distinguished. The first one, the famous dictum: to be is to be a value of variable, is the criterion of language ontological commitment; the second one which is the criterion of theory ontological commitments says that a theory is committed to an object (or objects of a given kind) if the object (the objects) must exist in order the theory to be true ${ }^{5}$. Needless to say existential theorems are responsible for such commitments. It is not very sharp and unambiguous distinction but nevertheless it helps to separate some issues. According to the first criterion Zalta's theory is committed to objects of Meinongian kind with respect to both modes of predication and, also, is committed to properties and relations. According to the second, the theory is committed to every particular Meinongian object, property, and relation that can be defined by formulas of the language employed (mainly by $\mathbf{A B O}$ and ABP).

\footnotetext{
5 These criterions are formulated for example in: [8], [9], [10]
} 


\section{A New Idea Concerning the Syntax}

The first problem one should face when formalising Meinongian ontology is a consistent treatment of incomplete and impossible objects. Usually adopted strategy is not that of choosing a many valued logic (or a logic of truth value gluts $)^{6}$. So, how can we render consistently sentences like $a$ is squared and $a$ is not squared in a formal two valued language? This can be done by introducing complex predicates which represent complex properties. Thus, we may have the negation of predicate is squared that is the complex predicate is not squared (or better: is non-squared). Our intention is that generally, the predication involving complex predicates (properties) would not be reducible to the predication involving only simple ones together with respective sentential connectives: the sentence $a$ is not squared would not be equivalent to the sentence it is not the case that a is silly for every $a$. It is an open question how many and what kinds of complex predicates and properties are to be at our disposal. But there is not doubt that we should have at least negations of predicates and properties since these are involved in the notions of incomplete and inconsistent objects. As a rule, complex predicates are expressed in formal language by an awkward lambda notation (cf. Parsons' and Zalta's theories) whereas the $\lambda$-elimination principle is suspended. However it is easy to notice that the primitive symbols of classical logic are sufficient for the purpose of expressing complex predicates. In particular, the negation of $P$ we can express simply by $x \neg P x$ instead of expressing it by $[\lambda x \neg P x]$. Generally, any expression of the form $x A$, where $A$ is a formula, will be a predicate expression. Subject-predicate sentences with the subject $a$ will be represented by formulas of the form: $a x A$. For example the following sentences will have the following logical forms respectively:

$\begin{array}{ll}\text { John is not silly } & a x \neg P x \\ \text { John is silly and lazy } & a x(P x \wedge Q x) \\ \text { John loves himself } & \text { axRxx } \\ \text { John loves Mary } & \text { axbyRxy } \\ \text { Mary is loved by John } & \text { byaxRxy } \\ \text { John loves somebody } & \text { ax } \exists y R x y \\ \text { Somebody is loved by John } & \exists y a x R x y\end{array}$

Of course, in this new syntax constants occupy the same positions as quantifiers in the classical syntax. That might look strange but equating cate-

${ }^{6}$ The only exception is Jacquette's theory in which Łukasiewicz three valued logic is applied. Cf. [3]. 
gorially names an quantifiers is not accidental here, it is not merely a kind of formal trickery. There exist good egsegetical and theoretical reasons for including quantifier or numerical objects in Meinongian ontology (together with the classical universal quantifier object). So not only are names and quantifiers of the same syntactic category but also they are of the same semantic category. Of course, there arises a problem whether the semantic relation between quantifiers and quantifier objects is a kind of reference at all but this is a different story.

\section{First System of Meinongian Objects}

Now let us describe more precisely a language for a theory of Meinongian objects, call it $\mathrm{M}_{1}$-language. Let us stress that we choose this language since it is generally more convenient to trace subsequent steps of ontological commitment.

\section{$\mathrm{M}_{1}$-language}

As we hinted earlier, the alphabet for $\mathrm{M}_{1}$-language consists of the same symbols as the alphabet of classical logic, i.e.:

(1) sentential connectives: $\neg, \supset$ (the other sentential connectives are introduced by means of usual definitions)

(2) the universal quantifier symbol: $\forall$

(3) the identity symbol: $=$

(4) individual variables: $x_{1}, x_{2}, \ldots$

(5) constants: $a_{1}, a_{2}, \ldots$

(6) predicate symbols: $P_{1}, P_{2}, \ldots$

(7) brackets: (,).

Symbols listed in (2) and (5) are called terms.

$\left(\mathbf{M}_{1}\right) \quad$ Let us assume that metavariables $s, t$ range over terms and $u, w, x, y, y_{1}, y_{2}, \ldots$ range over variables.

The grammar of $\mathrm{M}_{1}$-language is defined as follows: (a) every expression of the form $P y_{1} \ldots y_{n}$ where $P$ is $n$-argument predicate symbol, and every expression of the form $u=w$ is a formula; (b) if $A, B$ are formulas, then $\neg A$ and $(A \supset B)$ are formulas; (c) if $A$ is a formula then $x A$ is a predicate; (d) if $\Pi$ is a predicate and $t$ is a term, then $t \Pi$ is a formula ${ }^{7}$.

${ }^{7}$ We can define the set of formulas in the simpler way replacing conditions (c) and (d) by the single condition: if $A$ is a formula then $t x A$ is a formula. 
In $\mathrm{M}_{1}$-language Meinongian objects are represented exclusively by constants and as such they are not quantified. Their status resembles the status of what Quine calls virtual entities (classes, relations). In $\mathrm{M}_{1}$-language we have a rich category of predicate expressions; besides simple predicates there are complex ones, even those containing constants and quantifiers as their building blocks. Clearly, $\mathrm{M}_{1}$-language expresses only internal predications of M-objects; for instance we can express the sentence: Sherlock Holmes is a detective. The language can be equipped with various semantics and can give rise to various axiomatic systems. These may be classified with respect to the strength of closure conditions imposed on terms (Meinongian objects): starting from strong intensional systems in which objects (internally) possess exactly those properties from which they are constituted of (i.e. from tx $A$ no $t x B$ follows unless $A$ is identical to $B$ ), through intensional systems in which M-objects properties are closed under some entailment, and ending with systems in which objects properties are extensionally indistinguishable. Although extensional systems can hardly be considered as Meinongian theories by most prominent Meinongists ${ }^{8}$, they nevertheless obey the basic principles of Austrian philosopher's ontology. Besides, they have some essential advantages on their own. Let us present here first an extensional semantics and then an axiomatic system, which constitute in a sense the simplest logic based on $\mathrm{M}_{1}$-language, call it $\mathrm{M}_{1}$-logic. This particular choice of logic is almost unimportant for the general topic of our paper (we equally might consider an intensional version) but makes easier our discussion. Besides this extensional logic may be considered as a basic fragment of intensional ones.

\section{$\mathrm{M}_{1}$-semantics}

By a model of $\mathrm{M}_{1}$-language we mean a pair $\boldsymbol{M}=\langle D, I\rangle$ where $D$ is a non-empty set (called the domain of interpretation), $I$ is a function defined on terms and predicate symbols (called the interpretation):

(a) $\quad I(t) \subseteq \mathcal{P}(D)$, where $\mathcal{P}(D)$ is the power set of $D$ (in particular $I(\forall)=\{D\}$ );

(b) $\quad I(P) \subseteq D^{n}$, for $n$-argument predicate symbol $P$.

An assignment in $D$ is a function $V$ which assigns to every variable an element of $D$. Given $V$, by $V_{d}^{x}$ we mean the function which is just like $V$ with possible exception that $V_{d}^{x}(x)=d$.

\footnotetext{
${ }^{8}$ Only one expert in Meinong's philosophy, M. Schubert-Kalsi, admits that the philosopher would rather subscribe to extensional theory (personal communication).
} 
In $\mathrm{M}_{1}$-semantics truth conditions for atomic formulas, for negation and implication are the same as in the classical semantics. What is new in M-semantics is the truth condition for predication:

Formula $t x A$ is true in $M$ with respect to the assignment $V$ iff (*) there exists $X \in I(t)$ such that: $X \subseteq\left\{d \in D: A\right.$ is true in $M$ with respect to $\left.V_{d}^{x}\right\}$.

In particular, the formula $t x P x$ is true in $M$ iff there exists $X \in I(t)$ such that $X \subseteq I(P)$. Notice also that the condition retains the meaning of the universal quantifier with respect to the given interpretation.

A formula of $\mathrm{M}_{1}$-language is $\mathrm{M}_{1}$-valid iff it is true in every $\mathrm{M}$-model with respect to any assignment.

\section{$M_{1}$-system}

M-system is defined by the following axiom-schemata and rules of inference:

M1 Classical truth-functional tautologies;

M2 $\forall x(A \supset B) \supset(t x A \supset t x B)$;

M3 $A \supset \forall x A$, provided $x$ is not free in $A$;

M4 $\forall x A \supset A(u \mid x)$;

M5 txA $\supset \operatorname{ty} A(y \mid x)$;

M6 $u=u$;

M7 $u=w \supset(A \supset A(w \| u))$;

MP $\quad$ if $\vdash A \supset B$ and $\vdash A$, then $\vdash B$;

$\mathbf{G} \quad$ if $\vdash A$, then $\vdash \forall x A$.

One can notice that $\mathrm{M}_{1}$-logic closely resembles the classical first-order logic and is an extension of it - the classical axiomatics is explicitly included in the axiomatics of $\mathrm{M}_{1}$-system. What is worth emphasising is that the completeness of $\mathrm{M}_{1}$-logic easily follows from the completeness of first-order logic and the former logic is consistent as interpretable in a simple way in the latter. Moreover, compactness and Skolem-Löwenheim theorems hold for $\mathrm{M}_{1}$-logic as well. Despite these similarities, $\mathrm{M}_{1}$-logic has much stronger expressive power than the classical logic.

Let us comment briefly on the Meinongian character of $\mathrm{M}_{1}$-logic. According to $\mathrm{M}_{1}$-semantics, Meinongian objects are represented in the logic 
by sets of sets of individuals what is tantamount to extensional treating objects as sets of properties (see the definition of interpretation). A given object possesses a property iff the extension of the property is equal to or broader than the extension of some property constituting the object (see the condition $(*))$. It is easily seen that nonexistent objects even inconsistent and incomplete ones can be available in $\mathrm{M}_{1}$-semantic. For example, the round square is such an object when it is interpreted by the set: $\{$ set of circles, set of squares $\}$. The object in question possesses properties: being round, being a square, being a geometrical figure, but does not possess the property of having $1 \mathrm{~m}^{2}$ area and the property of not having $1 \mathrm{~m}^{2}$ area. Generally, for any set of properties there will be an object represented in the semantics by the set of extensions of these properties.

\section{Ontological Commitments}

If $\mathrm{M}_{1}$-language carries any ontological commitments then these coincide with the commitments of language of classical first-order logic. Individuals, i.e. members of the domain of interpretation, are exclusively quantified in objectual way in $\mathrm{M}_{1}$-logic (the mere fact that the universal quantifier is defined in $\mathrm{M}_{1}$-semantics like a generalised quantifier does not change its meaning). Consequently, only those Meinongian objects which can be identified with some individuals can be genuinely quantified (such an identification is expressible in $\mathrm{M}_{1}$-logic by the formula: $a x(x=y) \wedge \neg a x(x \neq y)$, which says that the individual $x$ is identical with the Meinongian object $a$ in the sense of sharing exactly the same properties ${ }^{9}$. What is specific for $\mathrm{M}_{1}$-logic however, is that quantification of (all) Meinongian objects may be simulated in a way. This is due to the validity of the following formulas:

$$
\begin{aligned}
& (\forall) \quad \forall x A \supset t x A, \\
& (\exists) \quad t x A \supset \exists x A,
\end{aligned}
$$

provided $t$ is a term with nontrivial interpretation, i.e. different from $\emptyset$ and $\{\emptyset\} .{ }^{10}$ These formulas mimic the classical universal instantiation and the existential generalisation respectively. Thus, we see then that $\mathrm{M}_{1}$-logic, as

\footnotetext{
9 The following formula is a theorem of $\mathrm{M}_{1}$-system: $a x(x=y) \wedge \neg a x(x \neq y) \supset(a x A \supset A(y \mid x))$.

10 Actually, $\mathrm{M}_{1}$-system theorems are only weaker formulas:

$t x B \supset(\forall x A \supset t x A)$,

$\neg t x B \supset(t x A \supset \exists x A)$.
} 
it stands, can be accepted even by someone who as Quine is particularly reluctant to nonexistent objects. ${ }^{11}$

So far we discussed only those commitments of $\mathrm{M}_{1}$-logic which have their source in the (interpreted) $\mathrm{M}_{1}$-language itself. Notice that in that logic no definition axiom for Meinongian objects has appeared yet although such axioms plays the crucial role in Parsons' and Zalta's Meinongian theories. As we have hinted earlier, definition axioms like $\mathbf{A B O}$ and $\mathbf{A B P}$, are usually responsible for system ontological commitments. Actually, $\mathrm{M}_{1}$-logic may be enriched with some definition schemata. These definitions enable us to introduce new terms on the basis of terms and predicates already present.

DM1 $[x A] x B \equiv \forall x(A \supset B)$, where $A$ contains at least one free variable $x$

DM2 $\hat{t} x A \equiv \neg t x \neg A$

(the inversion of term)

DM3 $(s \wedge t) x A \equiv s x A \wedge t x A$

(the conjunction of terms)

DM4 $(s \vee t) x A \equiv s x A \vee t x A$

(the disjunction of terms)

New terms receive appropriate interpretations in $\mathrm{M}_{1}$-semantics.

Now, for instance the object the round square can be explicitly defined on the ground of $\mathrm{M}_{1}$-language: [roundness] $\vee$ [squareness] and as such it is interpreted as $\{$ set of circles, set of squares\}. Also the existential quantifier can be defined as the inversion of the universal quantifier: $\hat{\forall}$, where $I(\exists)=\mathcal{P}(D) \backslash\{\emptyset\}$. Those definitions, especially DM2, DM2, DM4, resembles ordinary definitions of operations in the theory of "virtual classes" and are supposed to be equally innocuous from the ontological point of view. After all, these newly defined "objects" still remain unquantified and therefore, according to Quine's criterion, the definitions add no ontological commitment ${ }^{12}$.

Formulas $(\forall)$ and $(\exists)$ are unconditionally theorems of system which differs from $\mathrm{M}_{1}$-system only in that instead of $\mathbf{G}$ it has a stronger rule $\mathbf{M G}$ :

\footnotetext{
MG if $\vdash A$, then $\vdash t x A$ and $\vdash \neg t x \neg A$.

11 I argued elsewhere that $\mathrm{M}_{1}$-logic can be considered as a free logic. Cf. [7].

12 An essential difference between the ordinary theory of classes and our logic is that DM1-DM4 have the status of axioms and as such they need not be noncreative. For example, if these definitions are added to the system mentioned in the footnote 8 then we can simplify the rule MG: if $\vdash A$ then $\vdash t x A$. Anyway we are not going to discuss here the intricate problem whether the ontology of system is sensitive to such a creativity.
} 


\section{Second and Third System of Meinongian Objects}

Next let us consider $\mathrm{M}_{2}$-language which results from $\mathrm{M}_{1}$-language by allowing constants to occupy argument positions in formulas just as in the classical syntax, i.e. we assume:

Let us assume that metavariables $s, t$ range over terms

$\left(\mathbf{M}_{2}\right)$ and $u, w, y_{1}, y_{2}, \ldots$ range over variables and constants, $x$ and $y$ over variables.

instead of $\left(\mathbf{M}_{\mathbf{1}}\right)$ while letting remaining conditions of forming complex expressions be unchanged. Thus, for instance $P a, R x a$ are formulas of this new language. That means that it is now possible to express the external predication as well. For example, we may express in the language the sentence: Sherlock Holmes is a fictional character $(\mathrm{Pa})$ or even the sentence: Someone created Sherlock Holmes $(\exists x R x a) . \mathrm{M}_{2}$-language enable us to introduce a stronger system than $\mathrm{M}_{1}$-system, call it $\mathrm{M}_{2}$-system, which is based on the same axiom schemata but is extended in its applications in the usual way to constants. For example, as a particular case of $\mathbf{M} 4$ we have the axiom: $\forall x P x \supset P a$. Now Meinongian objects inhabit the same domain as other individuals ${ }^{13}$ but they are quantified only as subjects of external predication.

Clearly, when DM1-DM4 are present in $\mathrm{M}_{2}$-logic then the logic is theory committed to particular objects; for instance: $\exists x(x=[x A]), \exists x(x=\hat{t})$, $\exists x(x=(s \wedge t)), \exists x(x=(s \vee t))$ are theorems. But the ontological commitment of $\mathrm{M}_{2}$-logic is in a sense partial, i.e. only with respect to one mode of predication (this is of course a quite new case of ontological commitment not discussed by Quine). Needless to say, $\mathrm{M}_{2}$-logic is stronger as a formal system and richer in its expressive power than $\mathrm{M}_{1}$-logic. So it is not surprising that also its commitments are stronger. However, it is quite easy to prove that $\mathrm{M}_{2}$-logic is still consistent (with respect to $\mathrm{M}_{1}$-logic).

It is relatively easy to make a step further and extend $\mathrm{M}_{2}$-logic to a second order system bona fide. Like in the former case it is enough to modify (relax) the grammar rules: now we allow variables to occupy quantifier places as well. More precisely, we replace $\left(\mathbf{M}_{\mathbf{2}}\right)$ by $\left(\mathbf{M}_{\mathbf{3}}\right)$ :

$\left(\mathbf{M}_{3}\right) \quad$ Let us assume that metavariables $s, t, u, w, y_{1}, y_{2}, \ldots$ range over terms and variables; $x$ and $y$ over variables.

\footnotetext{
${ }^{13}$ We omit here details of semantics for the $\mathrm{M}_{2}$-language. Let us mention only that besides the interpretation of constants introduced in $\mathrm{M}_{1}$-semantics there will be another interpretation of constants which assigns them, when they appear in formulas on argument places, elements of the domain (like in the semantics of classical logic).
} 
Thus, for instance $y x A$, will be a formula of the new language, call it $\mathrm{M}_{3}$-language, and $y$ will be its free variable (but $x$ will not). Such a formula may be preceded by a quantifier expression $\exists y$ and this means that Meinongian objects are quantified as subjects of internal predication. For example, now we can formulate such sentences as for example: Sherlock Holmes is such that it internally possesses the property of 'being a detective' (axxyPy) or: There does not exist an object which possesses internally and externally a property of 'being a detective' $(\neg \exists x(x y P y \wedge P x))$. A new system, $\mathrm{M}_{3}$-system, may result when axiom schemata of $\mathrm{M}_{1}$-system are extended to $\mathrm{M}_{3}$-language (notions of free and bound variables and substitution are analogical to those of classical first order language). Unfortunately, such a system, as it stands (including axiom schemata), turns out to be inconsistent. To see this let us consider the object $[x y(x \neq y)]$ defined as follows:

$$
[x y(x \neq y)] x B \equiv \forall x(x y(x \neq y) \supset B)
$$

Let us abbreviate the term $[x y(x \neq y)]$ by $s$ and assume that:

(1) $\mathrm{s} x(s \neq x)$

(2) $\forall x(x y(x \neq y) \supset s \neq x)$

(3) $\quad s y(s \neq y) \supset s \neq s$

(4) $\neg s x(s \neq x)$ (from 3 and M6, M5)

Now let us assume that:

(1) $\neg s x(s \neq x)$

(2) $\exists x(x y(x \neq y) \wedge s=x)$

(3) $s x(s \neq x)$

(from 2 and M5)

Thus we get: $s x(s \neq x) \equiv \neg s x(s \neq x)$. Of course, we may safe the system while impoverishing its axiomatics, although — as it was mentioned in the case of Zalta's system - none of the known protection seems to be quite satisfactory from the philosophical point of view (even that which has been suggested for Zalta's system, for obvious reasons is not applicable here). Thus for example, we may weaken DM1 allowing only some stratified formula $\boldsymbol{A}$ to build a term. Such a modified $\mathrm{M}_{3}$-system appears to be consistent (at least relatively to the set theory) ${ }^{14}$. What is worth emphasising, it is a genuine second-order system. However, contrary to Zalta's system, the system is not committed to properties and relations. Surprisingly enough,

\footnotetext{
${ }^{14}$ The semantics for $\mathrm{M}_{3}$-systen is rather complicated and we are not going to consider it here.
} 
the quantification of objects implicitly comprises the quantification of properties. Roughly the idea is that in $\mathrm{M}_{3}$-logic some objects - as subjects of internal predication - can play the role of properties. Informally, the object a square rendered in $\mathrm{M}_{3}$-language by [squareness] and represented semantically by $\{$ set of squares $\}$ plays the role of property being a square ${ }^{15}$. Thus, we may conclude that Meinongian ontology need not to be committed to the ontology of properties and relations.

\section{Ten Magic Rings}

We have presented three systems of Meinongian objects starting with the weakest and ending with the strongest one. In $\mathrm{M}_{1}$-system no objects are quantified and therefore we may say that there is no genuine reference to Meinongian objects. Rather "objects" play here the same role as Quainian virtual objects. In $\mathrm{M}_{2}$-system external predication can be expressed and, at the same time, Meinongian objects are quantified but exclusively as subjects of this predication. Finally, in $\mathrm{M}_{3}$-system we have total quantification of these objects - both as subjects of internal and external predication. From the theoretical point of view there is possible a system in which Meinongian objects are quantified only as subjects of internal predication but this is not philosophically interesting case (we simply let variables to occupy quantifier places while forbidding constants to occupy argument positions).

One can wonder why we are distinguishing these systems, why we are not accepting just the strongest one while neglecting the weaker ones. Putting aside ones ontological likings and preferences, we wanted to show that the way of reference and the ontological commitment to Meinongian objects is

\footnotetext{
${ }^{15}$ The existence of objects that represent every property is secured in $\mathrm{M}_{3}$-logic by the theorem:

$$
\exists y(y x B \equiv \forall x(A \supset B)),
$$

which follows from the DM1. Now the following can also be proved in $\mathrm{M}_{1}$-logic:

$$
t x A \equiv t x \neg[x A] y(x \neq y) .
$$

This theorem has a stronger counterpart in $\mathrm{M}_{3}$-logic:

$$
\forall w \exists u(w x A \equiv w x \neg u y(x \neq y)) .
$$

It means that every (closed) formula $t x A$ is replaceable by a formula containing only terms and logical symbols (the negation and identity): $t x \neg s y(x \neq y)$. Since the structure of the latter formula is fixed we may write it in short: $t \epsilon s$, where $\epsilon$ will be a kind of ontological relation in Leśniewski's style. And this relation can simulate the relation of internal predication. That is why all we need in $\mathrm{M}_{3}$-logic is a definition axiom for objects.
} 
gradual and particular grades may correspond to different formal theories. Those theories reflect different phases of conscious apprehension of objects. So the first and the most important phase is such when an object presents to consciousness through nominal contents (we are conceiving objects through their internal properties). When we perform an act of reflection we are able to grasp an object of thought just as an object of thought (intentional object qua intentional) that is object in its external ontological structure. These two phases are rather pretheoretical but the next one is strongly theory laden. The sharp distinction between the first two and the third one is clearly reflected by the language. Everything that can be said in the natural language about objects of consciousness comprises the first two phases, although not without ambiguity as far as the internal-external predication distinction is concerned. To disambiguate the distinction we should resort to a formal language, say $\mathrm{M}_{2}$-language and the underlying logic. However, if we need to refer expressis verbis to the internal-external predication distinction we are forced to use in a substantial way a strong second order language and logic, e.g. $\mathrm{M}_{3}$-logic.

B. Russell claimed that the inconsistency of Meinong's ontology stems from the fact that the existent golden mountain is treated as an object, and consequently, it is both true that the objects exists and does not exist. Nowadays we know how to cope with such contradictions in a consistent way ${ }^{16}$. But, as we have seen, the inconsistency is still lurking in the contemporary Meinongian theories of strong expressive power (while the consistency of weaker theories is unquestionable). However the source of inconsistency is different then that pointed by Russell. The demonstrated paradoxes are typical paradoxes of size - these theories bring into existence too many objects $^{17}$.

Let us end this paper with an example of specific reference which Meinongian logics of the kind presented here might offer. There is widely known problem (or paradox) of "ten magic rings". This problem arises when one tries to treat fictional objects as Meinongian objects. Suppose we have a very short story: "There exists ten magic rings". So this story posits ten objects and each of them is constituted by the same properties: being magic, being a ring. Since objects constituted by the same properties are simply identical, actually there is exactly one "magic ring". Our solution is that

\footnotetext{
16 The existent golden mountain is existent in the sense of internal predication. But if we claim that it does not exist we mean the object in its external structure.

17 This problem is discussed by Parsons. Cf. [4], ch.9.
} 
the expression "ten magic rings" is semantically correlated with exactly one object however not with the object the magic ring but the ten magic rings. We have mentioned earlier that among other kinds of Meinongian objects there are numerical or quantifier objects and the ten magic rings is one of them. Also there is a room in Meinongian ontology for such objects as: one of ten magic rings, more than three magic rings, several magic rings etc.

\section{References}

[1] R. Clark, "Not Every Object of Thought Has Being: A Paradox in Naive Predication Theory", Noûs, Vol. 12, No. 2, 1978.

[2] K. Fine, "Critical Review of Parsons' Nonexistent Objects", Philosophical Studies 45, 1984.

[3] D. Jacquette; "Meinongian Logic. The Semantics of Existence and Non-existence" (to appear in Oxford University Press).

[4] T. Parsons, Nonexistent Objects, Yale University Press, 1982.

[5] K. Perszyk, Nonexistent Objects. Meinong and Contemporary Philosophy, Kluwer, Nijhoff 1993.

[6] J. Paśniczek, Meinongowska wersja logiki klasycznej. Jej zwiazki z filozofia języka, poznania, bytu i fikcji (in Polish: The Meinongian Version of Classical Logic. Its Relevance for the Philosophy of Language, Knowledge, Being, and Fiction), M. Curie-Skłodowska University Press 1988.

[7] J. Paśniczek, "Can Meinongian Logic be Free?", [in:] K. Lambert (ed.), New Directions in Free Logics (to appear).

[8] W. V. Quine, From the Logical Point of View, Harvard University Press, 1961.

[9] W. V. Quine, "Existence and Quantification", L'Age de la Science, 1, 1968.

[10] W. V. Quine, Philosophy of Logic, Prentice Hall, Englewood Cliffs, 1970.

[11] R, Routley, Exploring Meinong's Jungle and Beyond, Department Monograph \#3, Philosophy Department, Research School of Social Sciences, Australian National University, Canberra 1980.

[12] William Rapaport, "Meinongian Theories and Russellian Paradox", Noûs 12, 1978. 
[13] William Rapaport, "Nonexistent Objects and Epistemological Ontology", Grazer Philosophische Studien Vol. 25/26 1985.

[14] E. Zalta, Abstract Objects: An Introduction to Axiomatic Metaphysics, Dordrecht: Reidel 1983.

[15] E. Zalta, Intensional Logic and the Metaphysics of Intentionality, MIT Press, 1988.

JACEK PAŚNICZEK

Maria Curie-Skłodowska University

pl. Marii Curie-Skłodowskiej 4

20-031 Lublin, POLAND 